\title{
Absorption Enhanced Methanol Synthesis in a Trickle Bed Reactor over $\mathrm{Cu} / \mathrm{Zn} / \mathrm{Al}_{2} \mathrm{O}_{3}$ Catalyst
}

\author{
Peng Liu, Zhenmin Cheng* \\ State Key Laboratory of Chemical Engineering, East China University of Science and Technology, Shanghai \\ 200237, China \\ Email: zmcheng@ecust.edu.cn
}

Received April 2015

\begin{abstract}
Methanol synthesis in a trickle bed reactor with tetraethylene glycol dimethyl ether (TEGDME) as the liquid phase over a $\mathrm{Cu} / \mathrm{Zn} / \mathrm{Al}_{2} \mathrm{O}_{3}$ catalyst was investigated. The pressure was kept constant at $5.0 \mathrm{MPa}$, while the temperature ranged from $230^{\circ} \mathrm{C}$ to $260^{\circ} \mathrm{C}$ and the mass space velocity varied between $294 \mathrm{~L} \cdot \mathrm{Kg}^{-1} \cdot \mathrm{h}^{-1}$ and $1655 \mathrm{~L} \cdot \mathrm{Kg}^{-1} \cdot \mathrm{h}^{-1}$. The effects of temperature and space velocity on CO conversion and methanol productivity were studied. Methanol synthesis processes in trickle bed with the TEGDME and paraffin oil as liquid phase were compared with the fixed bed process. The results indicated that the optimal temperature was approximately $240^{\circ} \mathrm{C}$. When the space velocity was increased, the $\mathrm{CO}$ conversion decreased while the methanol productivity increased. The liquid introduced can help to keep the reactor nearly isothermal. For methanol synthesis in trickle-bed reactor, TEGDME was better than paraffin oil. Effect of TEGDME on the reaction was twofold. On one hand, it absorbs the methanol and speeds up the reaction. On the other hand, it also increases the mass transfer resistance and hinders the reaction.
\end{abstract}

\section{Keywords}

Absorption, Methanol Synthesis, Trickle Bed Reactor

\section{Introduction}

Methanol is one of the basic raw materials in various chemical applications and can also be used as a transportation fuel or a fuel additive. Conventionally, methanol is produced in gas-solid fixed bed reactor. Due to the highly exothermic and reversible nature of the reaction, methanol production rate is limited by the chemical equilibrium and the catalyst sensitivity to higher temperatures.

Sherwin and Frank [1] proposed a three-phase methanol synthesis process. In this process, the catalyst is suspended in an inert liquid, which can absorb the heat of reaction and keep the operation nearly isothermal. However, taking the upper limit of catalyst loading in the slurry liquid into consideration, the slurry reactor has to

\footnotetext{
${ }^{*}$ Corresponding author.
} 
operate at low space velocity. In addition, it also achieve lower conversion per pass ascribe to the high extent of backmixing. Kuczynski and Westerterp [2] suggested binding methanol with adsorbent in the gas-solid-solid trickle flow reactor (GSSTFR) to break the restriction of chemical equilibrium. In this reactor, the fine solid adsorbents trickle through the catalyst bed countercurrently to the syngas and adsorb the methanol selectivity as it formed. With this multifunctional reactor, they claimed that complete conversion of key reactant was attainable in single-pass operation. But, the solid circulation under high pressure is difficult on an industrial scale. In order to overcome this problem, Westerterp [3] employed a liquid absorbent instead of the solid adsorbent. They carried out the methanol synthesis reaction in a reactor system consisting of two packed bed in series with a methanol absorber between them. In the absorber, methanol is selectively absorbed from the product by tetraethyleneglycol-dimethylether (TEGDME) under reaction temperature. Their results revealed the per-pass conversion was high enough to avoid recycling of unconverted reactants. Pass [4] and Akgerman [5] developed a methanol synthesis technology in trickle bed reactor. Unfortunately, the liquid medium they adopted has lower solubility of methanol. Several investigators [6]-[8] have studied the methanol solubility in some solvent. These researches reveal the TEGDME exhibit good solubility of methanol.

None of the technologies aforementioned have solved the two problems (thermodynamic limitation and heat dissipation) involved in methanol synthesis simultaneously. The purpose of this study is to investigate the methanol synthesis in trickle bed reactor with TEGDME as the liquid medium. This process will also be compared with the fixed bed technology and trickle bed process with paraffin oil.

\section{Experimental}

\subsection{Comparison of the Liquid Mediums}

There are several generally accepted rules for selecting a solvent for a reaction, such as thermal stability, solubility of reactants and products, viscosity and specific heat capacity [9]. The main properties of TEGDME and paraffin oil are listed in Table 1. The viscosity was measured with a NDJ-8S rotating viscometer at $20^{\circ} \mathrm{C}$. It is shown that both the TEGDME and paraffin oil exhibit high enough boiling point to neglect their partial pressure in methanol synthesis process. Although the specific heat of TEGDME is lower than paraffin oil, it possesses lower viscosity and higher methanol solubility, which would decrease the mass transfer resistance, and hence widely used in solvent methanol process.

\subsection{Experimental Scheme}

A schematic diagram of the trickle bed reactor experimental setup is shown in Figure 1. The syngas (molar ratio

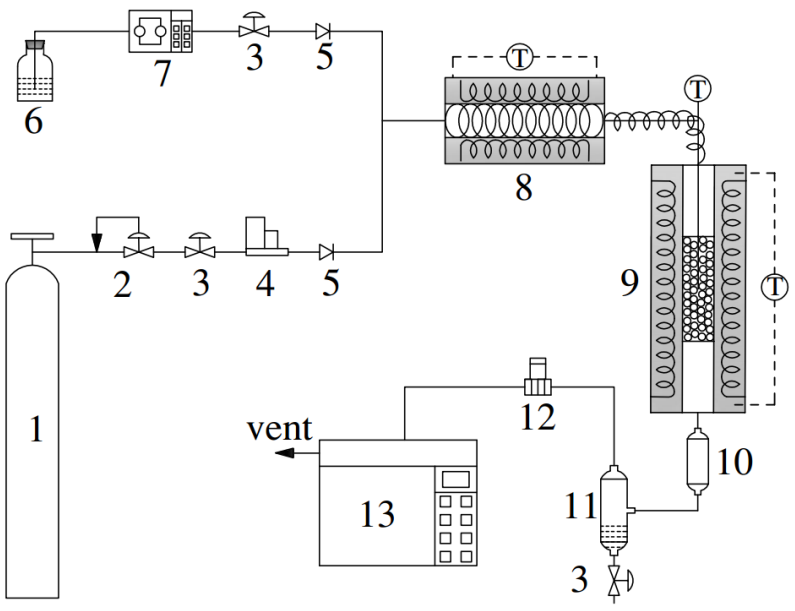

Figure 1. Schematic diagram of the apparatus for methanol synthesis in trickle bed reactor. 1: gas cylinder; 2: pressure regulator; 3 : ball valve; 4: mass flow meter; 5 : check valve; 6: liquid medium; 7: measuring pump; 8: preheater; 9: trickle bed reactor; 10: condenser; 11: separator; 12: back pressure regulating valve; 13: gas chromatography. 
Table 1. The properties of TEGDME and paraffin oil.

\begin{tabular}{ccccc}
\hline Medium & $T_{\mathrm{b}} /{ }^{\circ} \mathrm{C}$ & $\mu^{\mathrm{a}} / \mathrm{cp}$ & $C_{p} / \mathrm{J} \cdot \mathrm{g}^{-1} \cdot \mathrm{K}^{-1}\left(25^{\circ} \mathrm{C}\right)$ & $S^{\mathrm{b}} / \mathrm{mol}^{-\mathrm{m}^{-3}}$ \\
\hline TEGDME & 275 & 2.4 & $2.04[10]$ & $576[6]$ \\
Paraffin oil & $>340$ & 47.0 & $2.76[11]$ & $198[12]$ \\
\hline
\end{tabular}

a. measured at $20^{\circ} \mathrm{C}$. b. Temperature $=230^{\circ} \mathrm{C}$, methanol pressure $=0.50 \mathrm{MPa}$.

$\left.\mathrm{H}_{2}: \mathrm{N}_{2}: \mathrm{CO}: \mathrm{CO}_{2}=0.604: 0.163: 0.192: 0.041\right)$ was supplied by a cylinder. The gas flow rate was controlled and monitored by a mass flow controller, while the liquid medium was introduced by using a high-pressure flat-flow pump. The exit gas was cooled in an ice bath to condense the methanol and water, and then dried in a desiccant column. The vent gas was discharged down to atmospheric pressure by a pressure regulator and analyzed online by a gas chromatograph equipped with a TCD. In order to ensure complete wetting of the catalyst, the liquid rate in this study was maintained at $2.0 \mathrm{~mL} \cdot \mathrm{min}^{-1}$ [13]-[15]. About $10 \mathrm{~g}$ of catalyst was loaded in the reactor. Prior to the experiment, the $\mathrm{Cu} / \mathrm{Zn} / \mathrm{Al}_{2} \mathrm{O}_{3}$ catalyst was reduced in situ with a flow of $5 \% \mathrm{H}_{2}$ in $\mathrm{N}_{2}$ at $2.0 \mathrm{MPa}$ according to the following temperature program: start at room temperature, ramp at $2^{\circ} \mathrm{C} \cdot \mathrm{min}^{-1}$ to $160^{\circ} \mathrm{C}$ and then $1^{\circ} \mathrm{C} \cdot \mathrm{min}^{-1}$ to $280^{\circ} \mathrm{C}$, and keep at this temperature for $10 \mathrm{~h}$. After reduction the system temperature was adjusted to the desired reaction temperature.

\section{Results and Discussion}

\subsection{Effect of Reaction Condition on TEGDME Trickle Bed Reactor Methanol Synthesis}

\subsubsection{Effect of Temperature}

The effect of temperature on TEGDME trickle bed reactor methanol synthesis was investigated keeping the pressure of about 5.0 MPa, space velocity of $1110 \mathrm{~L} \cdot \mathrm{Kg}^{-1} \cdot \mathrm{h}^{-1}$ and TEGDME flow rate of $2.0 \mathrm{~mL} \cdot \mathrm{min}^{-1}$. Figure 2 indicates that as the temperature increase, both the conversion of $\mathrm{CO}$ and methanol productivity increase first and then decrease. It is undoubtedly correct as the methanol synthesis is a reversible exothermic reaction. The optimum reaction temperature is about $240^{\circ} \mathrm{C}$.

\subsubsection{Effect of Space Velocity}

Influence of the space velocity on TEGDME trickle bed methanol synthesis was conducted with the space velocity varied from $294 \mathrm{~L} \cdot \mathrm{Kg}^{-1} \cdot \mathrm{h}^{-1}$ to $1655 \mathrm{~L} \cdot \mathrm{Kg}^{-1} \cdot \mathrm{h}^{-1}$ while holding the system temperature about $240^{\circ} \mathrm{C}$, pressure about 5.0 MPa and TEGDME flow rate $2.0 \mathrm{~mL} \cdot \mathrm{min}^{-1}$. As shown in Figure 3, the CO conversion decreases while the methanol productivity increase when the space velocity increases. This can be attributed to that with the increase of space velocity, the residence time of reactant become shorter but the reaction rate will maintain at a faster level.

\subsection{Comparison of Different Methanol Synthesis Technologies}

\subsubsection{Temperature Fluctuation}

The reactor used in this study can be regarded as an adiabatic fixed bed, thus the temperature fluctuation in the reactor can characterize the heat dissipation capacity. Figure 4 compares the temperature fluctuation of different processes when the space velocity increased from $566 \mathrm{~L} \cdot \mathrm{Kg}^{-1} \cdot \mathrm{h}^{-1}$ to $1655 \mathrm{~L} \cdot \mathrm{Kg}^{-1} \cdot \mathrm{h}^{-1}$. It is clearly that the temperature fluctuation becomes significantly smaller when liquid is introduced into the reactor. Owing to the small scale reactor employed in this investigation, no obvious difference was observed between the TEGDME and paraffin oil trickle bed processes.

\subsubsection{Comparison of the Methanol Productivity}

Comparison of methanol productivity in different processes was illustrated in Figure 5. For trickle bed process, methanol productivity with TEGDME is markedly higher than that with paraffin oil. This can ascribe to the following two reasons: first, TEGDME possess lower viscosity than paraffin oil, which will favor the mass transfer process; second, the TEGDME can absorb the methanol as it produced and facilitate the reversible methanol synthesis reaction.

Figure 5 also indicates that methanol productivity in TEGDME trickle bed process is first higher and then lower than that in fixed bed reactor when the space velocity increase. This suggests the effect of TEGDME on 
methanol synthesis is twofold. On one hand, it absorbs the methanol in situ and speeds up the reaction. On the other hand, it increases the mass transfer resistance and decreases the reaction rate. When the space velocity is low, the reaction almost reaches the chemical equilibrium and the first effect is dominated, thus a higher produc-

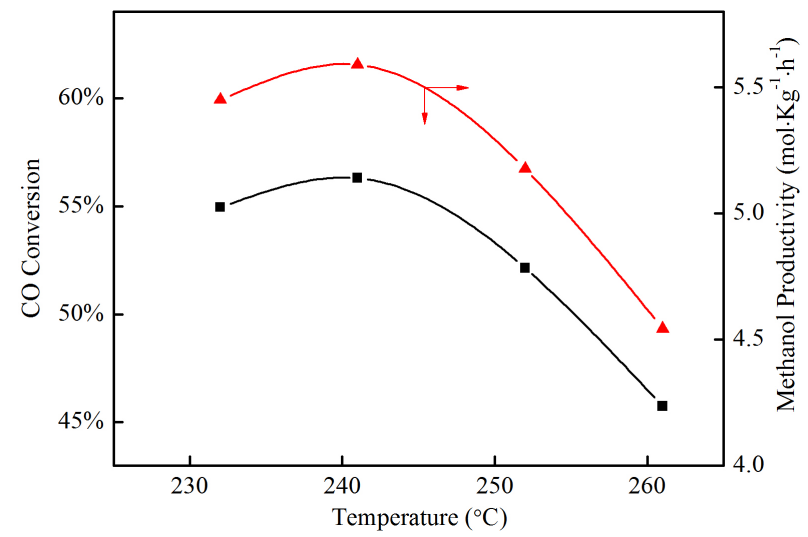

Figure 2. Effect of temperature on CO conversion and methanol productivity.

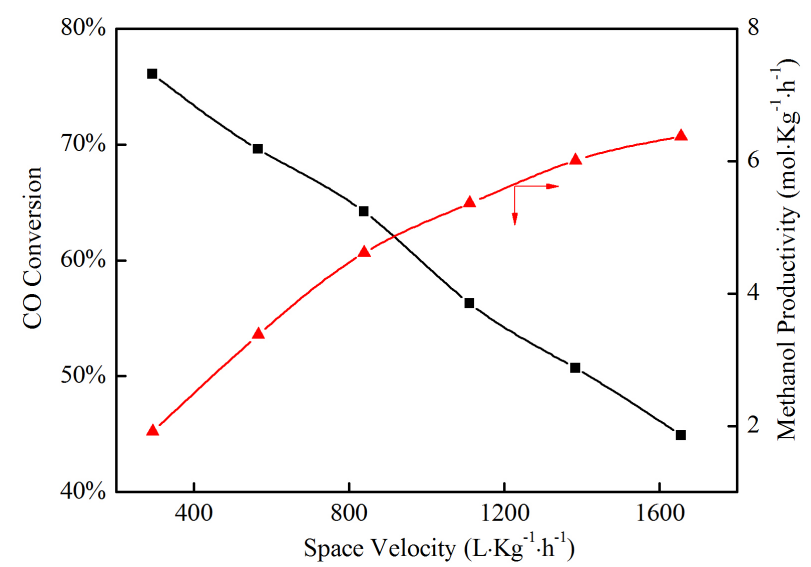

Figure 3. Effect of space velocity on CO conversion and methanol productivity.

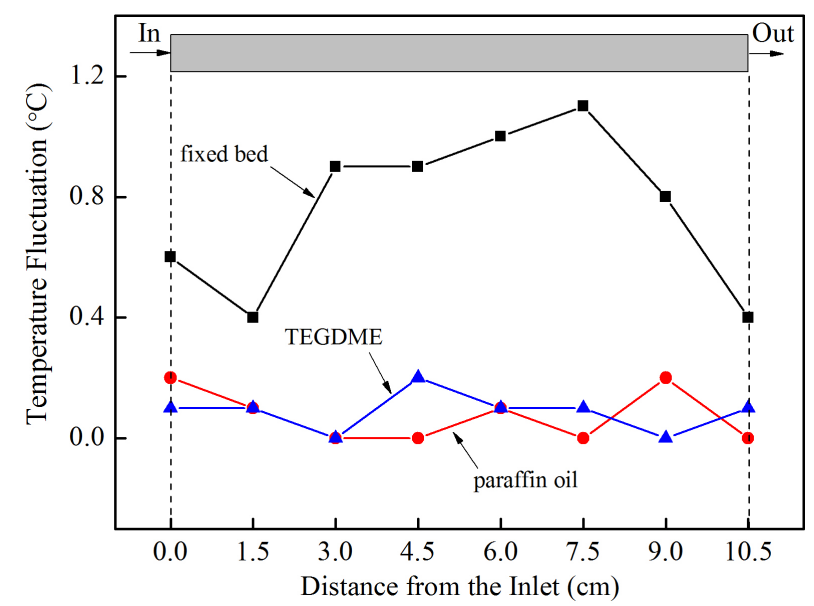

Figure 4. Comparison of temperature fluctuation in different processes. 


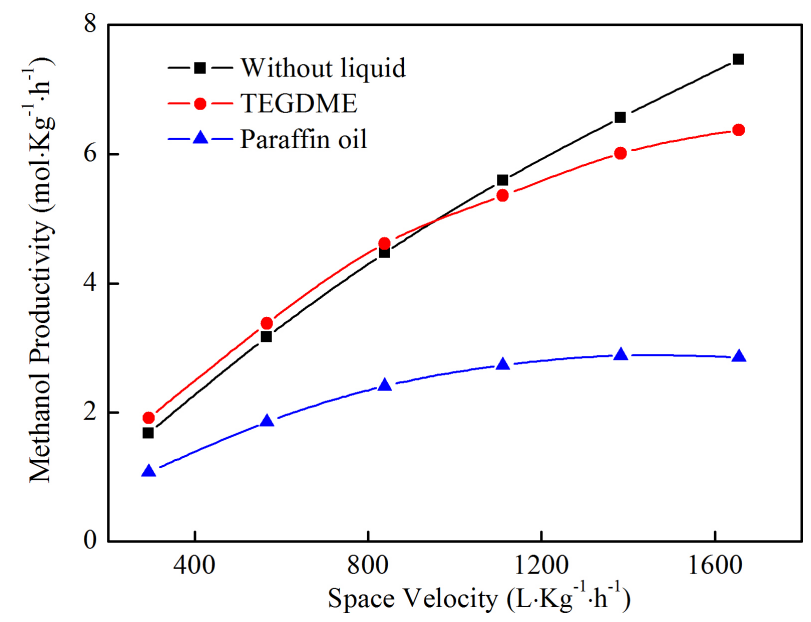

Figure 5. Comparison of methanol productivity in different processes.

tivity is achieved. With the increase of space velocity, the reaction departs from the equilibrium and the second effect gradually becomes the main aspect, thus causing the methanol productivity becomes lower than the fixed bed process.

\section{Conclusions}

(1) The TEGDME, which exhibits good solubility of methanol, is introduced into the trickle bed methanol synthesis process. For this process, the optimum temperature is about $240^{\circ} \mathrm{C}$. The $\mathrm{CO}$ conversion decreases while the methanol productivity increases as the space velocity increases.

(2) For trickle bed methanol synthesis process, methanol productivity with TEGDME is higher than that with paraffin oil. Effect of TEGDME on the reaction is twofold. On one hand, it absorbs the methanol as it formed and speeds up the reaction. On the other hand, it increases the mass transfer resistance and decreases the reaction rate. In addition, the liquid introduced can facilitate the heat dissipation and keep the system nearly isothermal.

\section{Acknowledgements}

The authors are grateful for the financial support of the Nature Science Foundation of China (21076072 and 21306045) and the Fundamental Research Funds for the Central Universities of China (222201314014).

\section{References}

[1] Sherwin, M.B. and Frank, M.E. (1976) Make Methanol by Three Phase Reaction. Hydrocarbon Process, 55, $122-124$.

[2] Kuczynski, M., Oyevaar, M.H., Pieters, R.T. and Westerterp, K.R. (1987) Methanol Synthesis in a Countercurrent Gas-Solid-Solid Trickle Flow Reactor. An Experimental Study. Chemical Engineering Science, 42, 1887-1898.

[3] Westerterp, K.R., Kuczynski, M. and Kamphuis, C.H.M. (1989) The Synthesis of Methanol in a Reactor System with Interstage Product Removal. Industrial \& Engineering Chemistry Research, 28, 763-771. http://dx.doi.org/10.1021/ie00090a018

[4] Pass, G., Holzhauser, C., Akgerman, A. and Anthony, R.G. (1990) Methanol Synthesis in a Trickle-Bed Reactor. AIChE Journal, 36, 1054-1060. http://dx.doi.org/10.1002/aic.690360712

[5] Tjandra, S., Anthony, R.G. and Akgerman, A. (1993) Low Hydrogen/Carbon Monoxide Ratio Synthesis Gas Conversion to Methanol in a Trickle Bed Reactor. Industrial \& Engineering Chemistry Research, 32, 2602-2607. http://dx.doi.org/10.1021/ie00023a024

[6] Kuczynski, M., Hart, W.'t. and Westerterp, K.R. (1986) Binary Vapour-Liquid Equilibria of Methanol with Sulfolane, Tetraethylene Glycol Dimethyl Ether and 18-Crown-6. Chemical Engineering and Processing: Process Intensification, 20, 60. http://dx.doi.org/10.1016/0255-2701(86)85008-5

[7] Graaf, G.H., Winkelman, J.G.M., Stamhuis, E.J. and Beenackers, A.A.C.M. (1988) Kinetics of the Three Phase Methanol Synthesis. Chemical Engineering Science, 43, 2161-2168. 
http://dx.doi.org/10.1016/B978-0-08-036969-3.50066-X

[8] Khosla, P., Krishnan, C., Elliott Jr., J.R. and Berty, J. (1991) Binary and Multicomponent Vapor-Liquid Equilibria of Synthesis Gas Components, Methanol and Water with Tetra Ethylene Glycol Dimethyl Ether (Tetraglyme). Chemical Engineering Communications, 102, 35-46. http://dx.doi.org/10.1080/00986449108910847

[9] Reichardt, C. (2003) Solvents and Solvent Effects in Organic Chemistry. 3rd Edition, Wiley-VCH Verlag, Weinheim. http://dx.doi.org/10.1002/3527601791

[10] Trejo, L.M., Costas, M. and Patterson, D. (1991) Effect of Molecular Size on the W-Shaped Excess Heat Capacities: Oxaalkane-Alkane Systems. Journal of the Chemical Society, Faraday Transactions, 87, 3001-3008. http://dx.doi.org/10.1039/ft9918703001

[11] Słoczyński, J., Grabowski, R., Kozłowska, A., Olszewski, P., Lachowska, M., Skrzypek, J. and Stoch, J. (2003) Effect of $\mathrm{Mg}$ and $\mathrm{Mn}$ Oxide Additions on Structural and Adsorptive Properties of $\mathrm{Cu} / \mathrm{ZnO} / \mathrm{ZrO} 2$ Catalysts for the Methanol Synthesis from CO2. Applied Catalysis A: General, 249, 129-138. http://dx.doi.org/10.1016/S0926-860X(03)00191-1

[12] Song, H.J., Zhang, H.T., Ying, W.Y. and Fang, D.Y. (2005) Solubilities and Volume Mass traNsfer Coefficients of Methanol and Steam in Liquid Paraffin. Petrochemical Technology, 34, 551-555.

[13] Colombo, A.J., Baldi, G. and Sicardi, S. (1976) Solid-Liquid Contacting Effectiveness in Trickle Bed Reactors. Chemical Engineering Science, 31, 1101-1108.

[14] Burghardt, A., Bartelmus, G., Jaroszyński, M. and Kołodziej, A. (1995) Hydrodynamics and Mass Transfer in a ThreePhase Fixed-Bed Reactor with Cocurrent Gas-Liquid Downflow. The Chemical Engineering Journal and the Biochemical Engineering Journal, 58, 83-99. http://dx.doi.org/10.1016/0923-0467(94)02956-3

[15] Cheng, Z.-M., Kong, X.-M., Zhu, J., Wang, Z.-Y., Jin, J. and Huang, Z.-B. (2013) Hydrodynamic Modeling on the External Liquid-Solid Wetting Efficiency in a Trickling Flow Reactor. AIChE Journal, 59, 283-294.

http://dx.doi.org/10.1002/aic.13785

\section{Nomenclature}

$C_{p}$ : specific heat, $\mathrm{J} \cdot \mathrm{g}^{-1} \cdot \mathrm{K}^{-1}$

$S:$ solubility, $\mathrm{mol} \cdot \mathrm{m}^{-3}$

$T_{\mathrm{b}}$ : boiling point, ${ }^{\circ} \mathrm{C}$

$\mu$ : viscosity, ср 